\title{
Growth and Characterization of Wide-Gap
} $\mathrm{Cd}_{1-x} \mathbf{Z n}_{x}$ Se Ternary Alloys by Using Electron Beam Evaporation Technique

\author{
J. Suthagar ${ }^{a}$, S. Rajesh ${ }^{a}$, K. Perumal ${ }^{b}$, T. Balasubramaniam $^{c}$ \\ AND N.J. Suthan KissingeR ${ }^{d, *}$ \\ ${ }^{a}$ Department of Physics, Karunya University, Coimbatore, India \\ ${ }^{b}$ Department of Physics, Sri Ramakrishna Mission Vidyalaya College of Arts \& Science, Coimbatore, India \\ ${ }^{c}$ Department of Physics, Kongunadu College of Arts \& Science, Coimbatore, India \\ ${ }^{d}$ School of Advanced Materials Engineering, Chonbuk National University, Jeonju-561 756, South Korea
}

(Received December 4, 2009; in final form January 29, 2010)

\begin{abstract}
$\mathrm{Cd}_{1-x} \mathrm{Zn}_{x}$ Se films with different zinc content were deposited by electron beam evaporation technique onto glass substrates for the application of solid-state photovoltaic devices. The structural, surface morphological and optical properties of $\mathrm{Cd}_{1-x} \mathrm{Zn}_{x}$ Se films have been studied in the present work. The host material, $\mathrm{Cd}_{1-x} \mathrm{Zn}_{x} \mathrm{Se}_{\text {, }}$ has been prepared by the physical vapor deposition method of electron beam evaporation technique under the pressure of $1 \times 10^{-5}$ mbar. The X-ray diffractogram indicates that these alloy films are polycrystalline in nature, of hexagonal structure with strong preferential orientation of the crystallites along $(002)$ direction. Linear variation of lattice constant with composition $(x)$ is observed. Surface roughness measured by atomic force microscopy is used to estimate the interface roughness. The optical properties show that the band gap $\left(E_{\mathrm{g}}\right)$ values vary from 2.08 to $2.64 \mathrm{eV}$ as zinc content varies from 0.2 to 0.8 . The surface morphological studies show the very small, fine and hardly distinguishable grains smeared all over the surface. The material properties would be altered and excellently controlled by adiusting the system composition $x$.
\end{abstract}

PACS numbers: 71.55.Gs, 61.05.cp

\section{Introduction}

II-VI polycrystalline semiconducting materials have come under increased scrutiny because of their wide use in the cost reduction of devices for photovoltaic applications. The interest in $\mathrm{Cd}_{1-x} \mathrm{Zn}_{x}$ Se ternary systems are increasing rapidly during the present decade, because the lattice constants and band gap values can be tuned systematically by incorporating various atomic constants of $\operatorname{Zn}(x)$. Such variations facilitate the development of new optoelectronic and electronic devices. These alloy systems are attractive semiconductor materials for light emitting devices in different colors (blue to red spectral region) [1].

The band gap of this material can be tuned from 1.70 to $2.70 \mathrm{eV}$ with $0 \leq x \leq 1$ [2]. It is an efficient absorber in the visible region of solar spectrum [3]. The applications of $\mathrm{Cd}_{1-x} \mathrm{Zn}_{x}$ Se in thin film devices like laser screen materials in projection color TV's $[4,5]$, nuclear radiation detectors [6], light emitting diodes, laser diodes,

\footnotetext{
* corresponding author; e-mail: suthanjk@gmail.com
}

electroluminescent and photoluminescent diodes, photovoltaics [7-12], etc. have shown its prominence and ability. The band structures, optical properties and crystal structures of both CdSe and ZnSe are very similar and therefore the system $\mathrm{Cd}_{1-x} \mathrm{Zn}_{x}$ Se would not only result in the feasibility of graded energy gap of a broad spectral sensitivity, but many more material characteristics can be altered and excellently controlled by the system composition $x$. Both CdSe and ZnSe are known to exist in either cubic zinc blende or hexagonal wurtzite crystal forms depending on the composition and the conditions of preparation. Compared to $\mathrm{CdSe}, \mathrm{Cd}_{1-x} \mathrm{Zn}_{x}$ Se is more stable and would replace $\mathrm{CdS}$ as a window material in solar cells. Further, the incorporation of ZnSe into CdSe has shown pronounced effect in enhancing the electrochemical power conversion efficiency [13-15].

The use of thin film polycrystalline semiconductors has attracted much interest in an expanding variety of applications in various electronic and optoelectronic devices. The technological interest in polycrystalline-based devices is mainly caused by their very low production costs. Different researchers [16-19] prepared $\mathrm{Cd}_{1-x} \mathrm{Zn}_{x}$ Se films 
by different techniques and studied their structural, optical and photoelectrochemical properties. In the present study we have prepared $\mathrm{Cd}_{1-x} \mathrm{Zn}_{x}$ Se films by electron beam evaporation technique at $100{ }^{\circ} \mathrm{C}$ with various zinc content incorporated with cadmium as $x=0.2,0.4$, 0.6 and 0.8 and their structural, optical and surface morphological properties were studied. All the films of $\mathrm{Cd}_{1-x} \mathrm{Zn}_{x}$ Se system were deposited under the same experimental conditions.

\section{Experimental procedure}

Thin films of $\mathrm{Cd}_{1-x} \mathrm{Zn}_{x}$ Se with different $\mathrm{Zn}$ composition were deposited from CdSe and ZnSe powder (Aldrich, 99.99\%) by electron beam (EB) evaporation technique using a HINDHIVAC Vacuum coating unit (model: 12 A4D) fitted with electron beam power supply (model: EBG-PS-3K). For the EB evaporation of $\mathrm{Cd}_{1-x} \mathrm{Zn}_{x}$ Se solid solutions, CdSe and ZnSe binary compounds are mixing in various atomic proportion and used as the precursor powder pellet for depositing the film. If the CdSe and ZnSe powder samples were heated using an electron beam collimated form, i.e. the d.c. heated tungsten filament cathode, the sample particles were spread out of the graphite crucible. For the improvement of homogeneous and uniform deposition on the substrate surface, the $\mathrm{Cd}_{1-x} \mathrm{Zn}_{x}$ Se pelletized targets have been utilized. Before making the pellets, the powder mixtures were ground in an agate mortar for $3 \mathrm{~h}$ to get uniformly mixed powders. Pellets were made at a pressure of 10 tons $/ \mathrm{cm}^{2}$ and heated at $100{ }^{\circ} \mathrm{C}$ for one hour. $\mathrm{Cd}_{1-x} \mathrm{Zn}_{x}$ Se films with $x=0,0.2,0.4,0.6,0.8$ and 1.0 were planned to be deposited on glass substrates, taking into consideration the different melting points of CdSe and $\mathrm{ZnSe}$ and vapor pressure of $\mathrm{Cd}, \mathrm{Zn}$ and Se elements. The different preparation parameters such as source-to-substrate distance $(12 \mathrm{~cm})$ and partial pressure $\left(10^{-5}\right.$ mbar) have been varied and optimized for depositing uniform, well adherent and transparent films. The films were found to be uniform, porous free and adhered well with the glass plates.

The crystalline size $(D)$ was calculated from the full width at half maximum (FWHM) $(\beta)$ by using the Scherrer formula

$$
D=\frac{0.94 \lambda}{\beta \cos \theta},
$$

where $\lambda$ is the wavelength of the $\mathrm{X}$-ray used, $\beta$ is the FWHM, $D$ is the particle size value, and $\theta$ is half the angle between the incident and the scattered X-ray beams. The strain values $(\varepsilon)$ can be evaluated by using the following relation:

$$
\varepsilon=\left(\frac{\lambda}{D \cos \theta}-\beta\right) / \tan \theta .
$$

The lattice spacing $(d)$ is calculated from the Bragg formula

$$
d=\frac{\lambda}{2 \sin \theta} .
$$

The lattice parameter $(a)$ is determined for the cubic structure by using the following expression:

$$
\frac{1}{d^{2}}=\frac{h^{2}+k^{2}+l^{2}}{a^{2}},
$$

where $h, k, l$ are the Miller indices of the lattice plane. The dislocation density $(\delta)$ has been calculated by using the formula for cubic ZnSe thin films

$$
\delta=\frac{15 \varepsilon}{a D} \text {. }
$$

The spectral normal transmittance $(T)$ was measured by UV-Vis-NIR spectrophotometer over the wavelength range $300-2500 \mathrm{~nm}$. The calculation of absorption coefficient $\alpha$ gives a higher value of $10^{4} \mathrm{~cm}^{-1}$ near the absorption edge and in the visible region. $\alpha$ depends on the radiation energy and on the composition of the films. The absorption data were analyzed using the relation for the near edge absorption of direct band-gap semiconductor films

$$
\alpha=K\left(h \nu-E_{\mathrm{g}}\right)^{1 / 2} / h \nu .
$$

The structural properties of the films were studied by the JEOL JDX X-ray diffractometer (XRD) using $\mathrm{Cu} K_{\alpha}$ radiation $(\lambda=1.5418 \AA)$ with $\mathrm{Ni}$ filter. The optical spectra were recorded in the wavelength range 300-2500 nm using UV-Vis-NIR spectrophotometer (Hitachi V-3400). Surface morphology of the films was studied by JEOL JSM-5610 L V (Japan) scanning electron microscope (SEM).

\section{Results and discussion}

The XRD patterns of the EB evaporated $\mathrm{Cd}_{1-x} \mathrm{Zn}_{x} \mathrm{Se}$ thin films were recorded to study the nature, phase and structure with different zinc $(x)$ content introduced into the CdSe matrix. Figure 1 shows the XRD spectra of $\mathrm{Cd}_{1-x} \mathrm{Zn}_{x}$ Se films with $x=0.2,0.4,0.6,0.8$. The sharp and well defined peaks indicate the polycrystalline nature of fabricated films. A high intense peak observed at $2 \theta=25.62^{\circ}$ corresponding to $(002)$ reflection and another peak very close to it at $2 \theta=24.18^{\circ} \mathrm{C}$ is assigned to $(100)$ reflection with a reduced intensity. The strong peak at $25.62^{\circ}$ confirms the presence of hexagonal structure for all the films with highly textured orientation along (0 02 ) plane. Further, $2 \theta$ values are found to shift towards higher angle side with increasing zinc content. This is a confirmative evidence of formation of homogeneous and alloyed $\mathrm{Cd}_{1-x} \mathrm{Zn}_{x}$ Se films by $\mathrm{EB}$ evaporation technique. Further, the presence of (0 0202$)$ preferred orientation indicates that the crystallites are oriented with their $c$-axis perpendicular to the substrate. The lattice space $d$ values agree well with the reported XRD data [20] for the $\mathrm{Cd}_{1-x} \mathrm{Zn}_{x}$ Se films deposited by electrochemical technique. For comparison the XRD spectra of CdSe and ZnSe were depicted in Fig. 1e,f. Table gives the lattice parameter values derived from XRD spectra for the $\mathrm{Cd}_{0.8} \mathrm{Zn}_{0.2} \mathrm{Se}, \mathrm{Cd}_{0.6} \mathrm{Zn}_{0.4} \mathrm{Se}, \mathrm{Cd}_{0.4} \mathrm{Zn}_{0.6}$ Se and $\mathrm{Cd}_{0.2} \mathrm{Zn}_{0.8} \mathrm{Se}$ thin films. 

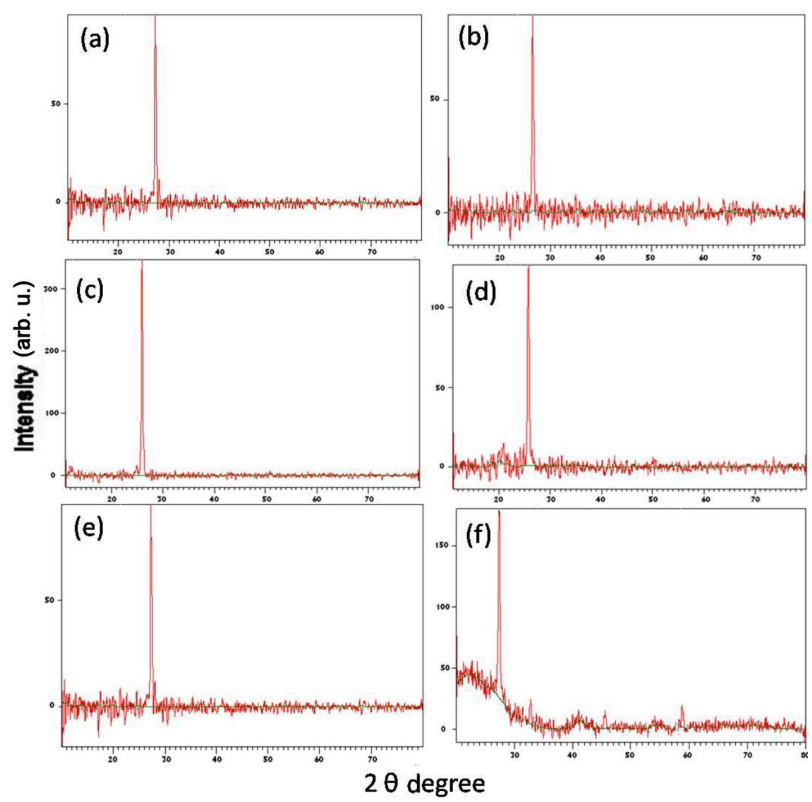

Fig. 1. XRD spectrum obtained for $\mathrm{Cd}_{1-x} \mathrm{Zn}_{x}$ Se films with different $x$ content: (a) $x=0.2$, (b) $x=0.4$, (c) $x=0.6$, (d) $x=0.8$, (e) and (f) XRD spectrum of CdSe and ZnSe.

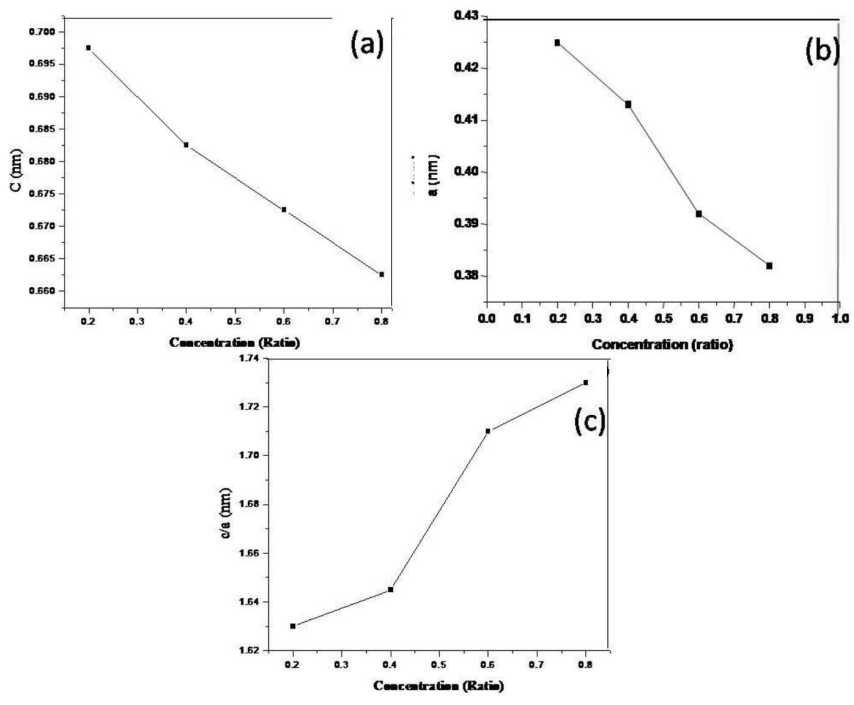

Fig. 2. Variation of lattice parameter (a) $c$ value with zinc concentration, (b) a value with zinc concentration, (c) $c / a$ value with zinc concentration.

TABLE

Structural parameters derived from XRD spectra for the $\mathrm{Cd}_{0.8} \mathrm{Zn}_{0.2} \mathrm{Se}, \mathrm{Cd}_{0.6} \mathrm{Zn}_{0.4} \mathrm{Se}$, $\mathrm{Cd}_{0.4} \mathrm{Zn}_{0.6}$ Se and $\mathrm{Cd}_{0.2} \mathrm{Zn}_{0.8}$ Se thin films.

\begin{tabular}{|c|c|c|c|c|c|c|}
\hline $\mathrm{Cd}_{1-x} \mathrm{Zn}_{x}$ Se film & $(h k l)$ & $2 \theta$ [deg] & $\begin{array}{c}d[\mathrm{~nm}] \\
\text { Experimental }\end{array}$ & $c[\mathrm{~nm}]$ & $a[\mathrm{~nm}]$ & $c / a[\mathrm{~nm}]$ \\
\hline \multirow[t]{2}{*}{$\mathrm{Cd}_{0.8} \mathrm{Zn}_{0.2} \mathrm{Se}$} & $\left(\begin{array}{lll}1 & 0 & 0\end{array}\right)$ & 24.17 & 0.3682 & 0.693 & 0.425 & 1.631 \\
\hline & $\left(\begin{array}{lll}0 & 0 & 2\end{array}\right)$ & 25.69 & $0.347(111)$ & & & \\
\hline \multirow[t]{2}{*}{$\mathrm{Cd}_{0.6} \mathrm{Zn}_{0.4} \mathrm{Se}$} & $\left(\begin{array}{lll}1 & 0 & 0\end{array}\right)$ & 24.87 & 0.358 & 0.682 & 0.413 & 1.651 \\
\hline & $\left(\begin{array}{lll}2 & 0 & 0\end{array}\right)$ & 26.51 & $0.337(111)$ & & & \\
\hline \multirow[t]{2}{*}{$\mathrm{Cd}_{0.4} \mathrm{Zn}_{0.6} \mathrm{Se}$} & $\left(\begin{array}{lll}1 & 0 & 0\end{array}\right)$ & 26.59 & 0.335 & 0.671 & 0.392 & 1.711 \\
\hline & $\left(\begin{array}{lll}2 & 0 & 0\end{array}\right)$ & 28.33 & $0.319(111)$ & & & \\
\hline \multirow[t]{2}{*}{$\mathrm{Cd}_{0.2} \mathrm{Zn}_{0.8} \mathrm{Se}$} & $\left(\begin{array}{lll}1 & 0 & 0\end{array}\right)$ & 26.95 & 0.331 & 0.658 & 0.384 & 1.713 \\
\hline & $\left(\begin{array}{lll}2 & 0 & 0\end{array}\right)$ & 28.49 & $0.311(111)$ & & & \\
\hline
\end{tabular}

The variation in lattice parameter with different $\mathrm{Zn}$ concentration is shown in Fig. 2. The lattice parameter values $c$ and $a$ were calculated on assuming hexagonal structure for all $\mathrm{Cd}_{1-x} \mathrm{Zn}_{x}$ Se films, given in Table. The value of $c$ decreased from 0.693 to $0.658 \mathrm{~nm}$ while that of $a$ decreased from 0.425 to $0.384 \mathrm{~nm}$ with increasing Zn content confirming the solid solution formation between CdSe and ZnSe binary compounds to make various ternary alloys. Further, the lattice parameters of these films follow the Vegard law which means that the lattice parameters of $\mathrm{Cd}_{1-x} \mathrm{Zn}_{x}$ Se ternary alloys, with zinc content $x$ can be linearly varied from the leading binary alloy CdSe. The slight variation in $c / a$ ratio was observed when the zinc concentration was increased from 0.2 to 0.8 . When the zinc concentration was increased from 0.2 to 0.8 , the $c / a$ ratio was increased from 1.63 to 1.73. Husain et al. [21] have reported the cubic structure for the whole range of zinc content from $x=0.2$ to 0.8 for their sintered films. This result predicts that these ternary alloys have hexagonal structure and that CdSeZnSe system forms excellent solid solutions in mixing by the exact substitution of $\mathrm{Zn}$ in the atomic locations of $\mathrm{Cd}$. 
Figure 3 shows the optical energy band gap spectrum of EB evaporated $\mathrm{Cd}_{1-x} \mathrm{Zn}_{x}$ Se films. The $E_{\mathrm{g}}$ values for our films vary from 2.08 to $2.64 \mathrm{eV}$ as zinc content varies from 0.2 to 0.8 . An increase in band gap is attributed to the mixing of higher band gap material, ZnSe with smaller band gap material CdSe. The variation of $E_{\mathrm{g}}$ is sublinear with zinc incorporation into the CdSe lattice. All the films are observed to have direct band gap transition, as that of the end components behave. The continuous variation of $E_{\mathrm{g}}$ with $x$ confirms the solid solution formation between CdSe and $\mathrm{ZnSe}$, so that their optical and electronic properties can be tailored. The variation of $E_{\mathrm{g}}(x)$ with increasing zinc content shows a sublinear behaviour.
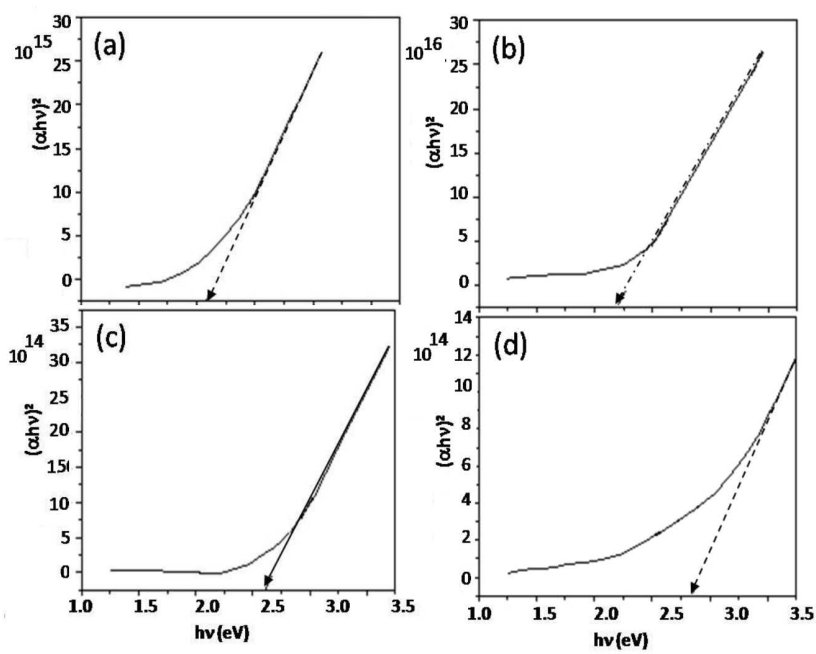

Fig. 3. $(\alpha h \nu)^{2}$ vs. $h \nu$ plot for $\mathrm{Cd}_{1-x} \mathrm{Zn}_{x}$ Se films with (a) $x=0.2$, (b) $x=0.4$, (c) $x=0.6$, (d) $x=0.8$.

The SEM pictures of $\mathrm{Cd}_{1-x} \mathrm{Zn}_{x}$ Se films on glass substrates are shown in Fig. 4. SEM is a promising technique for the topography study of samples, as it provides valuable information regarding the growth mechanism, shape and size of the particles and/or grains. The image of the deposited film reveals the uniform surface nature. Surface morphology by SEM studies shows very small, fine and hardly distinguishable grains smeared all over the surface. These are the characteristic features of zinc-rich surfaces. It is clearly observed from the surface morphological studies by scanning electron microscope that all the films have surfaces with uniform, homogeneous grain morphology. No crack was observed on the surface of the films. The sharp cleavage edge indicates the well adhesive nature of the films onto the substrates.

Thin films formed by EB evaporation technique, in the present study, with different zinc $(x)$ content have very interesting materials properties influenced by the presence of mixture structural phases, nanograins and different contents of $\mathrm{Cd}$ and $\mathrm{Zn}$. Hence, elemental analysis is an important study and the results obtained from energy dispersive X-ray (EDX) analysis are presented here. Figure $5 \mathrm{a}-\mathrm{d}$ shows the EDX spectra of $\mathrm{Cd}_{1-x} \mathrm{Zn}_{x}$ Se films

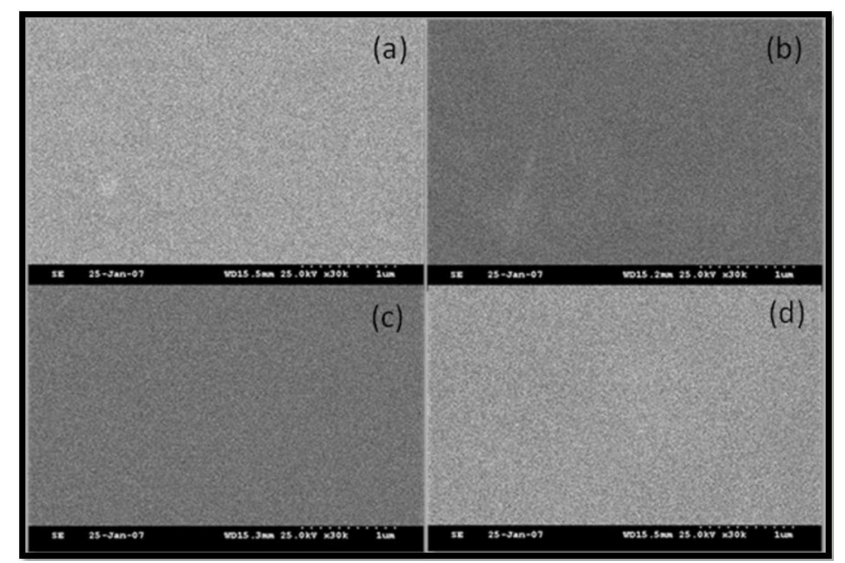

Fig. 4. SEM pictures taken for $\mathrm{Cd}_{1-x} \mathrm{Zn}_{x}$ Se films with (a) $x=0.2$, (b) $x=0.4$, (c) $x=0.6$, (d) $x=0.8$.

with zinc content $x=0.2,0.4,0.6$ and 0.8 , respectively. The films were deposited on glass substrates at $100^{\circ} \mathrm{C}$. It is found that the films are showing $\mathrm{Cd}$ and $\mathrm{Zn}$ content less than the percentage present in the starting material. However, the selenium content is always present in stoichiometric percentage and deficient. These results show the $n$-type nature of these solid solutions and excess metallic content over selenium reveals lower resistance.

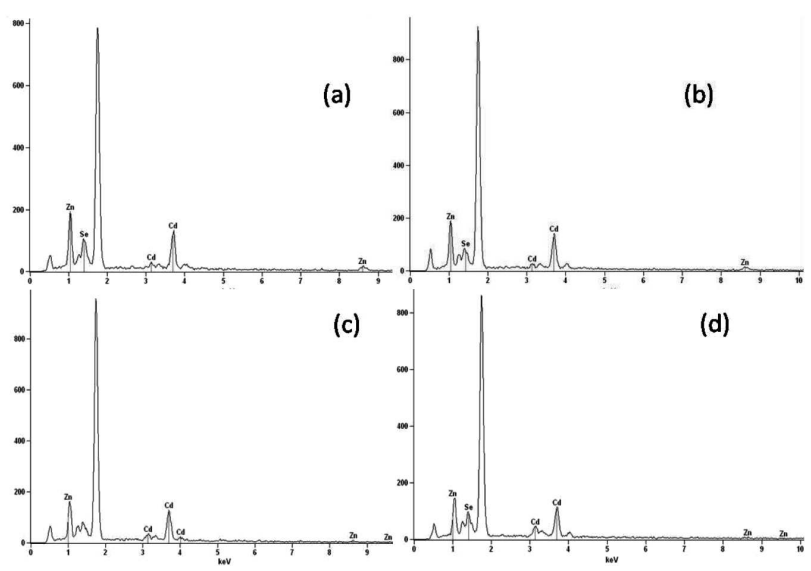

Fig. 5. EDX spectrum obtained for the $\mathrm{Cd}_{1-x} \mathrm{Zn}_{x} \mathrm{Se}$ films with $\quad$ (a) $x=0.2, \quad$ (b) $x=0.4, \quad$ (c) $x=0.6$, (d) $x=0.8$.

The two-dimensional (2D) atomic force microscopy (AFM) images are given in Fig. $6 \mathrm{a}-\mathrm{d}$ for the $\mathrm{Cd}_{1-x} \mathrm{Zn}_{x} \mathrm{Se}$ films with zinc content $x=0.2,0.4,0.6$ and 0.8 , respectively. It can be easily observed that the grain size decreases with increasing zinc content. This is acceptable because the atomic radius of $\mathrm{Zn}$ is $0.138 \mathrm{~nm}$, which is less than the atomic radius of $\mathrm{Cd} 0.171 \mathrm{~nm}$. The presently studied $\mathrm{Cd}_{1-x} \mathrm{Zn}_{x}$ Se film makes very good solid solution which means that zinc atoms replace cadmium atoms in the crystal lattice. This will obviously reduce the dimension of the lattice leading to the formation of linear grains 
with reduced grain size when one mixes to CdSe various amounts of ZnSe. For comparison, 2D AFM pictures are also given in Fig. 6e and f, respectively, for CdSe and ZnSe films. The calculation of average surface roughness $\left(R_{\mathrm{a}}\right)$ values for these films show very low values 0.12 , $0.13,0.20$ and $0.16 \mathrm{~nm}$, respectively. Such very low values predict the uniform surface of the $\mathrm{Cd}_{1-x} \mathrm{Zn}_{x}$ Se film prepared by the EB evaporation technique in the present study. It shows the compactness, pinhole free and well adherent nature of these films on glass substrates which will be very much useful for photoelectrochemical (PEC) devices. The line profile curves given under each 2D AFM pictures confirm these discussions.

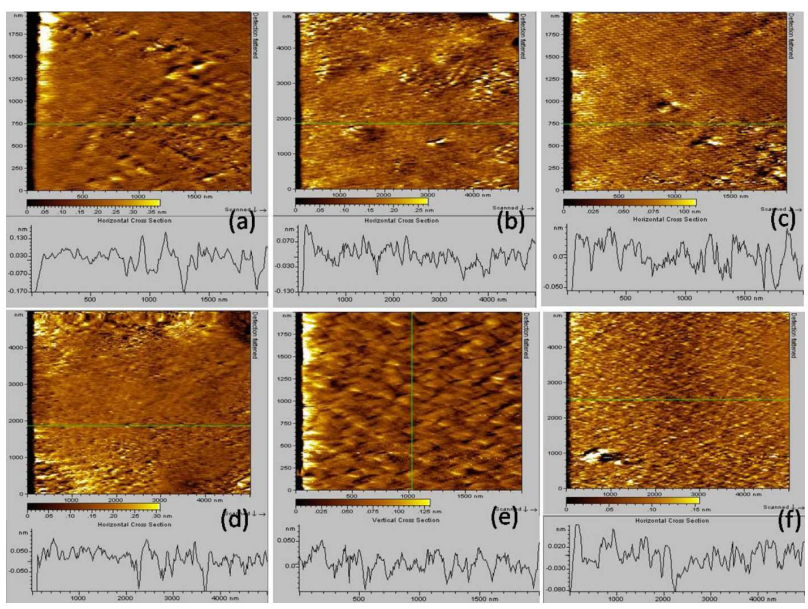

Fig. 6. AFM pictures (2D) taken for the $\mathrm{Cd}_{1-x} \mathrm{Zn}_{x} \mathrm{Se}$ films with (a) $\mathrm{Cd}_{0.2} \mathrm{Zn}_{0.8} \mathrm{Se}$, (b) $\mathrm{Cd}_{0.4} \mathrm{Zn}_{0.6} \mathrm{Se}$, (c) $\mathrm{Cd}_{0.6} \mathrm{Zn}_{0.4} \mathrm{Se}$, (d) $\mathrm{Cd}_{0.8} \mathrm{Zn}_{0.2} \mathrm{Se}$, (e) CdSe, (f) $\mathrm{ZnSe}$.

Figure $7 \mathrm{a}-\mathrm{d}$ shows the photoluminescence (PL) spectra recorded at room temperature for various $\mathrm{Zn}$ contents $0.2,0.4,0.6$ and 0.8 , respectively. The PL spectrum shifts towards higher energy values with increasing zinc content $x$, due to the increase of fundamental gap with $\mathrm{ZnSe}$ composition. There is no PL report on the full range of solid solutions with zinc content $x=0$ to 1.0. Toropov et al. [22] have the PL excitonic spectrum in superlattices (SLs) of CdSe insertions in a ZnSe matrix for elucidating the CdSe/ZnSe interface morphology. They have obtained a $\mathrm{PL}$ peak at $5 \mathrm{~K}$ for $\mathrm{Cd}_{0.23} \mathrm{Zn}_{0.77}$ Se single quantum well (QW) at about $2.77 \mathrm{eV}$ with $0.4 \mathrm{ML}$ (monolayer) and at $2.73 \mathrm{eV}$ with $1.15 \mathrm{ML}$. For our film with composition $\mathrm{Cd}_{0.2} \mathrm{Zn}_{0.8} \mathrm{Se}$, the peak is observed at about $2.65 \mathrm{eV}$ which is lower compared to ML films. It is natural that our films have thickness in the $200 \mathrm{~nm}$ where as ML layers of thickness are in the range about 4 to $12 \mathrm{~nm}$ and the effect of nanodimensional property comes into play. Similarly PL studies for multiply stacked CdSe quantum-dot (QD) arrays with a large ZnSe space thickness were conducted by Kirm et al. [23]. Their PL spectrum at $20 \mathrm{~K}$ showed a broad peak at $2.45 \mathrm{eV}$, which is related to interband transition from the ground electronic subband to the ground heavy hole subband $\left(E_{0}-H H_{1}\right)$ of the CdSe QDs. This value is found to be near to the PL peak at $2.41 \mathrm{eV}$ observed in the present work for the films $\mathrm{Cd}_{0.6} \mathrm{Zn}_{0.4} \mathrm{Se}$. Comparison of these results confirm that the $\mathrm{Cd}_{1-x} \mathrm{Zn}_{x}$ Se solid solution films deposited by EB evaporation technique in this study are of device quality nature and can be used for PEC solar cells and light emitting diode studies.

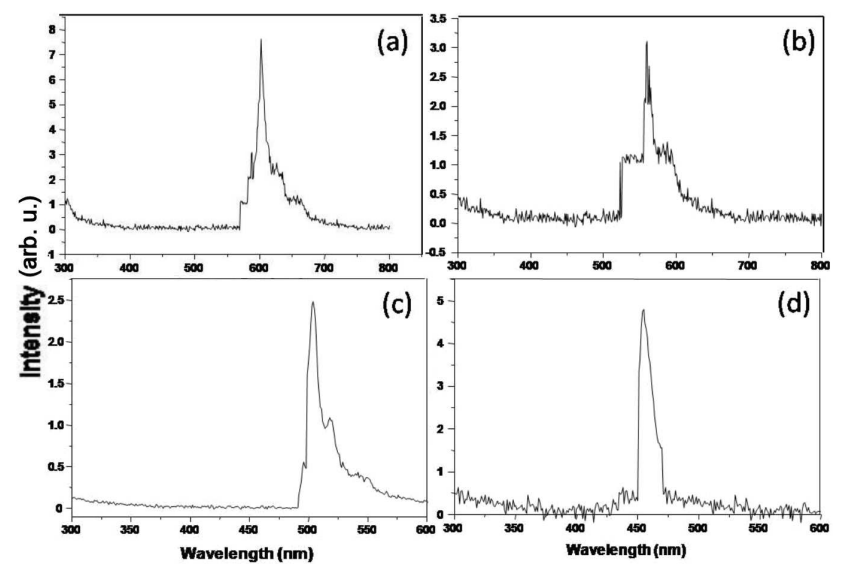

Fig. 7. PL spectrum obtained for the $\mathrm{Cd}_{1-x} \mathrm{Zn}_{x}$ Se films with $\quad$ (a) $x=0.2, \quad$ (b) $x=0.4, \quad$ (c) $x=0.6$, (d) $x=0.8$.

\section{Conclusion}

Ternary chalcogenide alloys of CdSe and ZnSe have been prepared by electron beam evaporation technique. In this paper we have described the detailed study of the structural, optical and surface morphological properties of $\mathrm{Cd}_{1-x} \mathrm{Zn}_{x}$ Se films prepared by electron beam evaporation technique. The X-ray diffraction analysis clearly shows the formation of predominant peaks along $\left(\begin{array}{lll}0 & 0 & 2\end{array}\right)$ growth orientation which exhibits that these alloy films are polycrystalline in nature and with hexagonal structure. The better aligned and highly oriented growth peaks enumerate the stoichiometric nature of the films. The XRD result predicts that these ternary alloys all have hexagonal structure and that CdSe-ZnSe system forms excellent solid solutions in all mixtures by the exact substitution of $\mathrm{Zn}$ in the atomic locations of Cd. The optical analysis reveals that the variation of $E_{\mathrm{g}}(x)$ with increasing zinc content shows a sublinear behavior, shows a lowering phenomenon in the alloy films of $\mathrm{Cd}_{1-x} \mathrm{Zn}_{x}$ Se. The nanostructural nature of $\mathrm{Cd}_{1-x} \mathrm{Zn}_{x}$ Se films is evident from the surface morphological results. We believe that these preliminary characteristic observations on the $\mathrm{EB}$ evaporated $\mathrm{Cd}_{1-x} \mathrm{Zn}_{x}$ Se films will be helpful to explore the device performance of the films for photoelectrochemical solar cells. 


\section{References}

[1] J. Ren, K.A. Boners, B. Sneed, D.L. Dreifus, J.W. Cook, J.F. Schetzin, R.M. Kolbas, Appl. Phys. Lett. 57, 1901 (1990).

[2] M. Husain, Beer Pal Singh, Sushil Kumar, T.P. Sharma, P.J. Sebastian, Sol. Energy Mater. Sol. Cells 76, 399 (2003).

[3] M. Kumar, M.K. Sharan, M. Sharon, Sol. Energy Mater. Sol. Cells 51, 35 (1998).

[4] K.C. Sharma, J.C. Garg, Indian J. Pure Appl. Phys. 26, 480 (1998).

[5] W. Lehmann, J. Electrochem. Soc. 113, 449 (1996).

[6] A. Burger, M. Roth, J. Cryst. Growth 70, 386 (1984).

[7] N.J. SuthanKissinger, M. Jayachandran, K. Perumal, C. Sanjeeviraja, Bull. Mater. Sci. 30, 547 (2007).

[8] A.S. Nasibov, Y.V. Korostelin, L.G. Susline, D.L. Fedorov, L.S. Markov, Solid State Commun. 71, 867 (1989).

[9] A.A. Bassam, A.W. Brinkman, G.J. Russel, J. Woods, J. Cryst. Growth 86, 667 (1988).

[10] N. Samarth, H. Luo, J.K. Furdyna, R.G. Alonso, Y.R. Lee, A.K. Ramdas, S.B. Quadri, N. Otsuka, Appl. Phys. Lett. 56, 1163 (1990).

[11] V. Krishnan, D. Hom, K.K. Misra, K. Rajeshwar, J. Electrochem. Soc. 139, 23 (1992).

[12] N.J. Suthan Kissinger, Natarajan Velmurugan, K. Perumal, J. Korean Phys. Soc. 55, 1577 (2009).
[13] G. Perna, V. Capozzi, M. Ambrico, V. Augelli, T. Ligonzo, A. Minafra, L. Schiavulli, M. Pallara, Appl. Surf. Sci. 233, 366 (2004).

[14] R. Chandramohan, C. Sanjeevraja, T.M. Mahalingam, Phys. Status Solidi A 163, 11 (1997).

[15] C. Natrajan, G. Nogami, M. Sharon, Bull. Electrochem. 12, 136 (1996).

[16] K.Y. Rajpure, S.M. Bamane, C.D. Lokhande, C.H. Bhonsale, Indian J. Pure Appl. Phys. 37, 413 (1999).

[17] U. Lunj, J. Kuhn, F. Goschenhofer, S. Einfeldt, C.R. Becker, G. Landwehr, J. Appl. Phys. 80, 6861 (1996).

[18] K.B. Kale, C.D. Lokhande, Appl. Surf. Sci. 253, 3109 (2007).

[19] D.R. Rao, R. Islam, Thin Solid Films 224, 191 (1993).

[20] R. Chandramohan, T. Mahalingam, J.P. Chu, P.J. Sebastian, Sol. Energy Mater. Sol. Cells 81, 371 (2004).

[21] R.B. Kale, C.D. Lokhande, Appl. Surf. Sci. 223, 343 (2004).

[22] A.A. Toropov, T.V. Shubina, S.V. Socokin, R.N. Kyutt, S.V. Ivanov, G.R. Pozina, J.P. Bergman, B. Monemar, M. Karlsteen, M. Willander, Appl. Surf. Sci. 166, 278 (2000).

[23] T.W. Kim, D.C. Choo, D.C. Lee, M. Jung, J.W. Cho, K.H. Yoo, S. Lee, K.Y. Seo, J.K. Furdyna, Solid State Commun. 122, 229 (2000). 